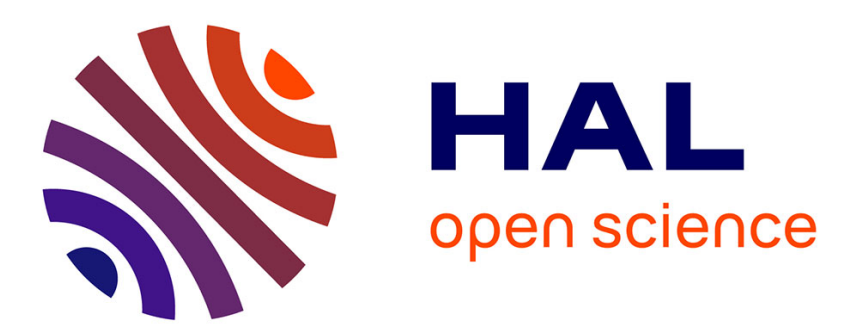

\title{
Reverse back-off mechanism for safety vehicular ad hoc networks
}

\author{
Razvan Stanica, Emmanuel Chaput, André-Luc Beylot
}

\section{To cite this version:}

Razvan Stanica, Emmanuel Chaput, André-Luc Beylot. Reverse back-off mechanism for safety vehicular ad hoc networks. Ad Hoc Networks, 2014, vol. 16, pp.210-224. 10.1016/j.adhoc.2013.12.012 . hal-01123827

\section{HAL Id: hal-01123827 \\ https://hal.science/hal-01123827}

Submitted on 5 Mar 2015

HAL is a multi-disciplinary open access archive for the deposit and dissemination of scientific research documents, whether they are published or not. The documents may come from teaching and research institutions in France or abroad, or from public or private research centers.
L'archive ouverte pluridisciplinaire HAL, est destinée au dépôt et à la diffusion de documents scientifiques de niveau recherche, publiés ou non, émanant des établissements d'enseignement et de recherche français ou étrangers, des laboratoires publics ou privés. 


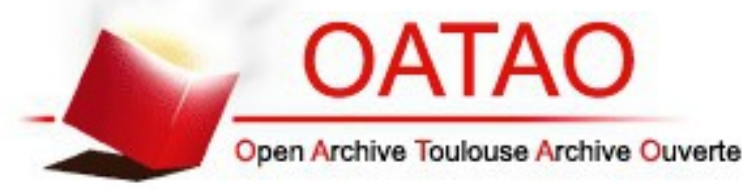

\section{Open Archive TOULOUSE Archive Ouverte (OATAO)}

OATAO is an open access repository that collects the work of Toulouse researchers and makes it freely available over the web where possible.

This is an author-deposited version published in : http://oatao.univ-toulouse.fr/ Eprints ID : 12855

To link to this article : DOI :10.1016/j.adhoc.2013.12.012 URL : http://dx.doi.org/10.1016/j.adhoc.2013.12.012

To cite this version : Stanica, Razvan and Chaput, Emmanuel and Beylot, André-Luc Reverse back-off mechanism for safety vehicular ad hoc networks. (2014) Ad Hoc Networks, vol. 16. pp. 210-224. ISSN $1570-8705$

Any correspondance concerning this service should be sent to the repository administrator: staff-oatao@,listes-diff.inp-toulouse.fr 


\title{
Reverse back-off mechanism for safety vehicular ad hoc networks
}

\author{
Razvan Stanica $^{\mathrm{a}, *}$, Emmanuel Chaput $^{\mathrm{b}}$, André-Luc Beylot ${ }^{\mathrm{b}}$ \\ a Université de Lyon, INSA Lyon, CITI-INRIA UrbaNet, F-69621, Villeurbanne, France \\ ${ }^{\mathrm{b}}$ Université de Toulouse, INP Toulouse, ENSEEIHT, IRIT IRT, 31000, Toulouse, France
}

\begin{abstract}
A B S T R A C T
Vehicular ad hoc networks can play an important role in enhancing transportation efficiency and improving road safety. Therefore, direct vehicle-to-vehicle communications are considered as one of the main building blocks of a future Intelligent Transportation System. The success and availability of IEEE 802.11 radios made this technology the most probable choice for the medium access control layer in vehicular networks. However, IEEE 802.11 was originally designed in a wireless local area network context and it is not optimised for a dynamic, ad hoc vehicular scenario. In this paper, we investigate the compatibility of the IEEE 802.11 medium access control protocol with the requirements of safety vehicular applications. As the protocols in this family are well-known for their scalability problems, we are especially interested in high density scenarios, quite frequent on today's roads. Using an analytical framework, we study the performance of the back-off mechanism and the role of the contention window on the control channel of a vehicular network. Based on these findings, we propose a reverse back-off mechanism, specifically designed with road safety applications in mind. Extensive simulations are carried out to prove the efficiency of the proposed enhancement scheme and to better understand the characteristics of vehicular communications.
\end{abstract}

\section{Introduction}

Despite the efforts invested by the automotive industry in the design of on-board safety systems, traffic accidents remain the number one cause of death in the age group between 1 and 44 in both Europe and the United States [1]. This is mainly due to the fact that, until recently, these safety systems took a reactive approach, trying to reduce the consequences, but not the number of road accidents.

Direct vehicle-to-vehicle (V2V) communications promise to open up an entire class of proactive approaches in the transportation world, becoming a very interesting research topic in both industry and academia. This interest was further increased by the October 1999 US Federal

\footnotetext{
* Corresponding author. Tel.: +33 472437311.

E-mail address: razvan.stanica@insa-lyon.fr (R. Stanica).
}

Communications Commission's (FCC) decision to allocate $75 \mathrm{MHz}$ of spectrum in the $5.9 \mathrm{GHz}$ band for Intelligent Transportation Systems (ITS) uses. In 2008, the European Commission also assigned $30 \mathrm{MHz}$ of spectrum in the same band for ITS purposes (with a possible extension to $50 \mathrm{MHz}$ ).

More than a decade after the FCC's decision, the automotive industry seems to be ready for the next step: adding wireless communications capabilities inside vehicles and creating a vehicular ad hoc network (VANET) with the role of enhancing road safety.

The maturity and availability of IEEE 802.11 compliant products meant this technology was the logical choice for the medium access control (MAC) and physical (PHY) layers in VANET. Soon after the 1999 FCC decision, the American Society for Testing Materials (ASTM) took up the task of developing a communication standard for direct 
short-range communications (DSRC) in the $5.9 \mathrm{GHz}$ band, based on the already successful IEEE 802.11a standard. In 2004, the IEEE decided to improve the ASTM standard and integrate it in a wider architecture for Wireless Access in Vehicular Environments (WAVE), and created the 802.11 Task Group p (TGp) to this effect. TGp reached its goal in July 2010, when the IEEE 802.11p amendment was published [2].

However, a solution based on IEEE 802.11 also has its contesters, especially because of the scalability problems that characterise the protocols from this family in ad hoc scenarios [3]. As a consequence, in its European ITS Communication Architecture [4], the ETSI adopted IEEE 802.11p at the MAC layer, but also left the door open for evolutions of the standard, or even for other technologies.

The main problem stems from the fact that the IEEE 802.11 standard has been designed with a wireless local area network (WLAN) context in mind, and it struggles to cope with the challenges of ad hoc communications. Moreover, the vehicular environment raises unique challenges, and therefore the protocol needs to cope with variable node density, important mobility, high relative speeds, driver privacy and a communication range of up to $1 \mathrm{~km}$, all of this while being used by applications with an important impact on human lives.

The broadcast nature of vehicular safety communications is another issue affecting the performance of IEEE 802.11 p in a VANET. Broadcast messages receive a special treatment by the Distributed Coordination Function (DCF) that provides medium sharing in IEEE 802.11, as they are not acknowledged and do not use the Request-to-Send (RTS) and Clear-to-Send (CTS) messages. This further increases the scalability problem, as the DCF is practically stripped from all the mechanisms related to congestion control, to a basic Carrier Sense Multiple Access (CSMA) scheme.

The standardisation bodies acknowledged this problem, and the ETSI released a set of technical specifications focused on decentralised congestion control for ITS operating in the $5 \mathrm{GHz}$ band [5]. This framework proposes three different mechanisms for congestion control: beaconing frequency adjustment, adaptive data rate, and transmission power control. These are generic solutions, that could work for any MAC protocol, and they are not specifically designed with IEEE 802.11 in mind.

In this paper, we study the impact of the contention window $(C W)$, used by the IEEE 802.11 back-off mechanism, under different values of vehicular density. This is a parameter well-known for its central role in congestion control in WLANs [6], but slightly neglected in V2V scenarios. We begin by providing a theoretical analysis of IEEE 802.11 in a vehicular context. This allows us to distinguish important characteristics of the safety messages, and compare the performance of the MAC layer using different sets of parameters. With these properties in mind, we propose a new back-off mechanism for safety VANETs, the reverse back-off mechanism, and we study its performance through extensive simulations.

Our main contributions can be therefore divided in two groups:
- We propose an analytical framework that accurately models the behaviour of an IEEE 802.11-based MAC protocol in a vehicular network. We show that the broadcast and periodic nature of the safety messages have an important impact on the optimal value of the contention window. We study the relationship between vehicular density and $C W$ and we find that it is different from the dependence exhibited by a classical WLAN scenario.

- Based on these findings, we propose the reverse back-off mechanism. In this approach, the contention window adaptation is no longer based on collided messages, but on the expiration of periodic safety messages. We use a realistic simulation framework to study this solution from the perspective of different metrics. We also discuss the different reasons for a lost message in a vehicular environment, and show that the reverse back-off mechanism does not only increase the reception probability, but also strongly modifies the loss distribution in a positive way for safety applications.

The remainder of this paper is organised as follows. Section 2 presents background information on vehicular safety communications, VANET congestion control, and contention window adaptation in IEEE 802.11. Section 3 gives the details of the analytical model and discusses numerical results for a high density vehicular network. The reverse back-off mechanism is described in Section 4, while its evaluation makes the object of Section 5. Finally, Section 6 concludes the paper.

\section{Background}

This section discusses the special characteristics of vehicular safety communications, as specified by the standardisation bodies. It also highlights previous work in the area of congestion control in vehicular networks and regarding possible adaptations of the contention window in IEEE 802.11

\subsection{Vehicular safety communications}

In both Europe and US, the spectrum allocated for vehicular communications has been divided in $10 \mathrm{MHz}$ channels. From these channels, one is known as the control channel $(\mathrm{CCH})$ and it is used solely by road safety applications. The rest of the channels, called service channels $(\mathrm{SCH})$ can be used by both safety and non-safety applications. In this paper, we address medium access control using IEEE 802.11p on the control channel, therefore we are only interested in safety scenarios.

The number of proposed vehicular safety applications that could use direct V2V communication is impressive [7]. However, at a close inspection, it can be noticed that all these applications practically use the same information, coming from on-board sensors of neighbouring vehicles: speed, acceleration, steering angle, location, etc.

Considering this, the standardisation bodies decided to add a supplementary layer between the applications and the transport protocol. The role of this layer, called message 
sublayer in the WAVE architecture and facilities layer in the ETSI terminology, is to keep an accurate image of the surrounding environment inside every vehicle, and to provide applications with the desired information.

The facilities layer only needs two types of messages in order to achieve these objectives, called (in the ETSI ITS architecture) Cooperative Awareness Message (CAM) and Decentralised Environmental Notification Message (DENM). CAMs are regular beacons, transmitted by every station with a predetermined frequency, and containing details about the vehicle that might be relevant to its neighbours from a safety point of view. On the other hand, if a vehicle detects a potential hazard (e.g. a sudden brake) and it considers that this information needs to be quickly disseminated to the other traffic participants, it transmits a DENM.

However, regardless the scenario and message type, these safety messages are always transmitted in broadcast mode at the MAC layer. Even in the case when the transmitted information targets a certain geographical area (e.g. an electronic brake alarm is only interesting for vehicles travelling in the same direction with the transmitter and situated behind it), the message is still broadcast and the filtering happens at the facilities layer, as described by the ETSI framework [8].

The broadcast nature of the VANET control channel is an essential property, that distinguishes it from other IEEE 802.11-based networks. As a matter of fact, the numerous studies on DCF usually focus on unicast traffic, and broadcast messages are only considered for control purposes. Oliveira et al. quantify the influence of broadcast traffic on the performance of IEEE 802.11 networks [9], and they find out that the effect of broadcast messages becomes significant when the proportion of broadcast traffic is higher than $50 \%$. In this scenario, the behaviour of the network largely deviates from what is predicted by classical DCF models. However, the authors consider this situation quite unreal and they do not further investigate the issue.

Another important characteristic of safety messages comes from the limited lifetime of CAMs. As these beacons are produced periodically by the facilities layer, there is a certain probability that they can expire before the MAC layer has the opportunity to transmit them. When a CAM is waiting for the IEEE 802.11 back-off timer to expire, and the next beacon also arrives in the transmission queue, the first message has to be dropped, as its transmission would only disseminate outdated information to its neighbours. While dropping an expired safety message from the MAC layer queue is a requirement of the ETSI ITS architecture, the IEEE WAVE framework provides no explicit recommendation on this issue. However, we believe that the dissemination of outdated information is not only a waste of network resources, but also a danger from a safety point of view. Therefore, we assume in this work that safety messages have a limited lifetime and they are dropped when the expiration time is reached. As we will show in the next sections, this property, rarely taken into consideration in other studies, has a significant effect on the optimal value of different MAC layer parameters.

In this paper, we focus on the delivery of safety beacons in the one-hop neighbourhood of a vehicle. The reception ratio and the inter-reception time of CAMs represent essential performance metrics for multiple envisioned vehicular safety applications, such as Intersection Collision Warning, Lane Change Assistant, or Emergency Vehicle Warning.

\subsection{Congestion control}

IEEE $802.11 \mathrm{p}$ radios can communicate at distances of $1 \mathrm{~km}$. In a simple scenario, with a two-lane road in both directions and an average inter-vehicular distance of $50 \mathrm{~m}$ (a medium density highway), the number of onehop neighbours reaches 160 vehicles. This is clearly a more challenging environment than the classical WLAN, with a central access points and no more than 10-20 nodes. The MAC layer protocol therefore needs solutions for this congested environment in order to achieve scalability.

Several ideas appeared in this sense in the literature. The first one is focused on reducing beaconing frequency in congested scenarios [10]. The idea behind this mechanism is that, when the number of vehicles increases, their speed decreases and the distance travelled during a beaconing period reduces. The vehicles can therefore transmit the CAMs with a frequency based on their speed. However, while this could bring an important load reduction, the solution is incompatible with several safety applications, where low speeds or stationary vehicles are common without necessarily being the result of increased road traffic (e.g. left-turn assistant). Other studies propose to adapt the beaconing frequency based on the measured channel busy ratio (CBR) or some other indicator of channel quality [11]. However, the use of the CBR as an estimator for the vehicular density is only accurate for low vehicular densities. Once the vehicular density is important enough, the CBR remains stable at a value around 0.9 even if more nodes are added to the network. Using the results provided in [12], we estimate that, in a scenario similar to ours, the CBR allows distinguishing only between vehicular densities lower than $100 \mathrm{veh} / \mathrm{km}$. Moreover, trying to keep the channel quality above a certain threshold by reducing the CAM frequency leads to very large beaconing periods (more than $3 \mathrm{~s}$ in [11] for a target CBR of 0.7).

A second solution is based on data rate control: in a high-density network, the vehicles use a more complex modulation and a higher data rate [13]. This reduces the duration of a transmission and allows more vehicles to access the channel. However, while theoretically interesting, this approach seems to be difficult to put into practice. A very detailed experimental study by Bai et al. [14] shows that the V2V channel is noisy, containing a high number of grey zones, zones with intermediate probability of message reception. According to these results, Quadrature Phase-Shift Keying (QPSK), with an associated data rate of $6 \mathrm{Mb} / \mathrm{s}$, is the only feasible modulation in this environment. Increasing the data rate to $18 \mathrm{Mb} / \mathrm{s}$, for example, leads to a reception ratio of less than $50 \%$ at $100 \mathrm{~m}$ from the transmitter. Considering that these tests were conducted with only two vehicles and a low level of interference, it is reasonable to expect that data rate adaptation will have a very limited use in vehicular networks and that it cannot represent a solution for congestion control. 
Probably the most investigated mechanism in this context is transmission power control [15], where vehicles in a dense region use a lower transmission power, achieving a better spatial reuse. This technique reduces the reception probability at higher distances from the transmitter, while keeping a high reception ratio for close neighbours. This is a very useful property for safety applications, where the impact of geographically close vehicles is much more important than the one of vehicles situated several hundred metres away. However, as we will show in Section 5, adjusting the transmission power cannot solve the scalability problem on its own and needs complementary mechanisms.

All the solutions described above are protocol agnostic, and they were integrated in the ETSI ITS decentralised congestion control framework [5]. Nevertheless, other mechanisms focused on IEEE 802.11 can be imagined. One of the main parameters of the CSMA protocols is the carrier sense threshold. As discussed in [16], the optimal value of this threshold varies with the vehicular density, and an adaptive mechanism can bring a significant improvement in beaconing reception ratio.

\subsection{Contention window in IEEE 802.11}

Another mechanism with a central role in IEEE 802.11 is the binary exponential back-off (BEB). In DCF, a node that finds the medium occupied when it attempts a transmission must randomly choose a back-off time in order to reduce the probability of a collision. This back-off time is given by an integer number, uniformly chosen between 0 and the contention window used by the BEB mechanism. This translates into the choice of a random number of idle slots that the node must wait before starting the transmission. As long as the channel is detected as busy, the backoff timer is blocked and the countdown restarts when the medium has been idle for the duration of a DCF Interframe Space (DIFS). Finally, when the timer expires, the node directly transmits the message. In unicast mode, the sender waits to receive an acknowledgement (ACK) message from the receiver. If this does not happen, the $C W$ is doubled and the transmission procedure is relaunched. However, this is not the case if the message is broadcast, as ACKs are not used in this situation because of the imminent collisions they would cause. Therefore, in broadcast mode, the message is transmitted only once, using the minimal value of the contention window $\left(C W_{\min }\right)$.

The impact of $C W_{\min }$ on the performance of the MAC layer has been thoroughly studied in the case of WLANs. Even before the release of the first version of the IEEE 802.11 standard, Bianchi [6] showed that the optimal value for the minimal contention window depends on the number of contending stations. More exactly, his analysis shows that, in a saturated WLAN, the throughput is maximised when:

$C W_{\min } \approx n_{c} \sqrt{2 T_{t}}$

where $n_{c}$ is the number of nodes in the network and $T_{t}$ is the time needed to transmit the message (acknowledgement included). Building on these results, Cali et al. [17] determined that the protocol's performance peaks when the time the channel is idle due to the back-off mechanism equals the time the channel is occupied by collisions $\left(T_{\text {idle }}=T_{\text {col }}\right)$.

Despite this well known property, the IEEE 802.11 standard does not include any mechanism for the adjustment of $C W_{\min }$ when the number of contending stations grows. There are several reasons behind this choice:

- The protocol was designed to work in wireless LANs, with a central access point and a limited number of client stations in mind.

- The RTS/CTS handshake limits collisions to the short control messages and therefore the time the channel is busy due to collisions decreases. This implies that, for an optimal functioning, $T_{\text {idle }}$ also needs to be reduced, which requires a lower contention window.

- The massive success of multimedia applications imposed a small $C W_{\min }$ in order to minimise the delay experienced by sensitive video and voice services. A reduced contention window improves the MAC layer performance in this case, while the BEB mechanism is there as a back-up for the cases when the number of contending stations increases.

However, these properties are no longer true in a VANET, where the node density is highly variable and a station can go, within a few minutes, from a very sparse environment to several hundred contending neighbours. Adding to this the fact that the RTS/CTS handshake is not implemented and the BEB mechanism is deactivated by the lack of ACK messages, none of the properties that allowed the use of a small contention window in IEEE 802.11 WLANs holds in this scenario.

For more than a decade, all the IEEE 802.11 enhancements related to $C W_{\min }$ adaptation in mobile ad hoc networks belonged to one of two categories. The methods in the first class (e.g. [18]) estimate the number of contending stations in the two-hop neighbourhood and use Eq. (1) or some variants to calculate the optimal contention window. The second type of mechanisms consider the overhead introduced by the local density estimation as prohibitive, and the amount of time the channel is sensed idle and the number of collisions are instead measured. The contention window is adjusted in this case in order to keep valid the equality $T_{i d l e}=T_{\text {col }}$ : when there are too many collisions on the channel, the back-off time (and with it the idle time) is increased, while a channel idle for long time durations results in a reduced contention window. A notable example from this second class is IdleSense, proposed by Heusse et al. [19].

Nevertheless, recently, Jiang and Walrand [20] took a totally different approach concerning the back-off mechanism in CSMA networks, proposing optimal CSMA (oCSMA). The idea behind this new protocol is to adapt the contention window of a node as a function of its queue length. In oCSMA, a node begins with an initial value for contention aggressiveness (which can be easily translated in a certain $C W_{\min }$ ) and, when the number of messages in a link queue increases, the transmitter becomes more aggressive in the competition for channel access by 
reducing the value of $C W_{\min }$. In spite of having very low complexity and requiring only local information, oCSMA has been proven to achieve throughput optimality under both continuous-time and discrete-time back-off duration [21].

The solutions described in a vehicular environment also belong to the two categories discussed above. Rawat et al. [22] propose a heuristic based on the number of detected collisions, where the contention window is increased if the number of collided messages is higher than a predefined threshold. Another solution is proposed in [13] where, in an initial phase, the local node density is estimated and directly used in Eq. (1). The value of the contention window is further refined by increasing $C W_{\min }$ when the percentage of lost beacons becomes higher than a target packet error ratio.

A rapid analysis of the compatibility between these solutions and the safety VANET shows that the design of these mechanisms does not take into account the characteristics of the $\mathrm{CCH}$. First of all, the Bianchi relationship is true for a unicast saturated one-hop WLAN cell, while a safety vehicular network uses broadcast and is neither saturated nor fully connected. Second, because ACKs are not used, collisions remain difficult to detect in V2V communication, therefore IdleSense and other similar approaches (e.g. [22]) cannot be directly transposed in a vehicular environment. Finally, as discussed above, expired beacons are dropped. This implies that the MAC layer always has at most one safety message to transmit, and the queue length cannot determine the contention window as proposed in oCSMA.

From our knowledge, the only contention window adaptation mechanism taking into account the expiration probability of safety beacons is the one proposed by Di Felice et al. [23]. The authors propose a two-phase adaptation: in a first step, the contention window increases with the channel busy ratio while, in a second time, the back-off value is refined based on the time remaining before message expiration. However, this solution is designed to solve the problem of message accumulation at the beginning of a $\mathrm{CCH}$ period in the case of channel switching between service and control channels. In this case, the back-off time is computed for all enqueued CAMs at the beginning of the $\mathrm{CCH}$ period, a different scenario from the one considered in this paper, where a radio is always switched on the control channel and the contention time is computed as soon as the message reaches the MAC layer, in agreement with current radio hardware. Moreover, a mechanism such as the one proposed in [23] assumes the channel busy ratio is a good estimator of the vehicular density, a property which is not true in high density scenarios, as discussed above.

Also in a vehicular context, a lot of effort has been dedicated to the design of back-off mechanisms that would optimise the delivery of messages, especially DENMs, over a certain geographical area $[24,25]$. In this form of multihop broadcast, the objective is to set the smallest backoff time to the best forwarding nodes, in order to minimise the information propagation delay. However, this problem is fundamentally different from the single-hop broadcast optimisation we focus on.
The reverse back-off mechanism proposed in this paper is the first solution we are aware of that takes into account the properties of single-hop safety beaconing. We show that the Bianchi model is not accurate when message expiration is considered, and we no longer use failed transmissions as a trigger for contention window adaptation. Similarly to oCSMA, the mechanism uses a relatively high value for the contention window, but the buffer status is not a parameter used when adapting the back-off time.

\section{System model}

The complexity of a VANET, where vehicular traffic and special network properties need to be considered, makes the analytical study of MAC layer performance in a vehicular environment. Previously proposed unicast frameworks based on Markov chain analysis have been extended to a vehicular context by Ma et al. [26] and Vinel et al. [27], but fail to take into account essential properties of the safety beaconing, such as the limited lifetime of the messages. The impact of expired messages is not considered even in some models specifically designed for the performance analysis of vehicular safety applications (e.g. $[28,29])$.

The two studies we are aware of that take into account the expiration probability of safety beacons are those described by Campolo et al. [30] and Di Felice et al. [31]. However, both these studies focus on single radio devices that need to periodically switch between the control channel and one service channel, and the beaconing expiration is the result of such a switching. Unlike these analytical frameworks, the one we propose considers a radio that permanently remains on the $\mathrm{CCH}$, as foreseen by the ETSI ITS architecture. While the ongoing debate between the proponents of the two functioning modes is out of the scope of this paper, we would like to point out that the two systems are highly different from a MAC layer point of view, hence the need for a different analytical model.

Moreover, it is noteworthy that both [30,31] make much stronger assumptions than the model proposed in the present work. Two important examples are the hypothesis that all the vehicles are in each other's transmission range (meaning that hidden nodes are ignored), and the requirement that the communication range is equal to the carrier sense range. Neither of these properties holds in reality and, as shown by our analysis, the impact of hidden nodes is quite important and cannot be ignored.

The model described in this chapter takes a different approach, concentrating on mean values observed during a beaconing period and, despite making some simplifying assumptions, it proves to be a decent tool that can help assess the impact of different MAC layer parameters.

Throughout this study, we consider all the vehicles use the same values for parameters relevant to MAC layer congestion control, with the exception of the contention window. This means that the beaconing frequency, the transmission rate and power, or the receiver sensitivity are the same for all nodes. While this assumption does not hold in practice, it allows us to focus in this work on 


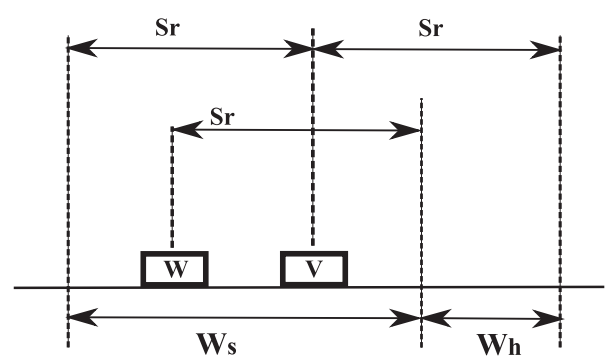

Fig. 1. Different zones around the tagged vehicle $V$.

the impact of the back-off mechanism on the reception of vehicular safety messages.

\subsection{Busy slot probability}

Let us choose one vehicle $V$ from the network, with a carrier sense range $S_{r}$, as shown in Fig. 1. Considering a mean vehicular density of $\lambda$ veh $/ \mathrm{km}$, node $V$ has $n_{c}$ onehop neighbours with:

$E\left[n_{c}\right]=2 \lambda S_{r}$.

We consider that all the nodes have similar parameters (same carrier sense range, same transmission power), and we focus on a generic neighbour $W$ of the tagged node $V$. $W$ is situated somewhere in the carrier sense range of $V$, either in front or behind.

Considering a beaconing period consisting of $N_{T}$ slots, let us assume vehicle $V$ will sense as busy, on average, $E\left[N_{b}\right]$ from these slots. In this case, the busy slot probability seen by vehicle $V$ can be expressed as:

$P_{b}=\frac{E\left[N_{b}\right]}{N_{T}}$.

Ideally, because of the periodic nature of safety beaconing, the number of busy slots would only be given by the mean number of sensed stations $\left(E\left[n_{c}\right]\right)$, and the duration of a beacon in slots $\left(N_{s}\right)$. However, in a real scenario, some of the neighbours experience collisions (with probability $\left.P_{c o l}\right)$, while others do not transmit during a given beaconing period because their safety messages expire (with probability $\left.P_{\text {exp }}\right)$. In this case, $E\left[N_{b}\right]$ can be calculated as follows:

$E\left[N_{b}\right]=E\left[n_{c}\right] N_{s}-E\left[n_{c}\right] P_{\exp } N_{s}-E\left[n_{c}\right] P_{\text {col }} \frac{E\left[N_{c o l}\right]}{E\left[n_{i}\right]}$

where $N_{\text {col }}$ is the number of slots occupied by a collision and $n_{i}$ is the number of nodes involved in the collision.

This equation can be solved for any values of $N_{\text {col }}$ and $n_{i}$, for example for experimentally determined values. In the simulation study described in Section 5 , we found $E\left[n_{i}\right]=2.11$, meaning that, in the vast majority of cases, there are only two nodes involved in a collision, an intuitive result in the case of a road constrained vehicular topology. Based on these results, in the following we make the simplifying assumption of $n_{i} \approx 2$, meaning that there are two situations capable of producing a collision at node $V$. In the first case, the collision takes place between two nodes that are in the carrier sense range of one another but start transmitting simultaneously, in the same slot (with probability $P_{c S}$ ). The duration of the collision in these circumstances is equal to the duration of a beacon, $N_{s}$. The second possibility is that the colliding stations are hidden from each other, and the two CAMs can therefore superpose with probability $P_{c h}$ at node $V$ on a number of slots uniformly distributed between 1 and $N_{s}$.

It is important to understand that, in this model, a collision does not necessarily imply a lost message, but only the simultaneous reception of more than one signal by node $V$. Using the capture effect or advanced decoding techniques, some of these messages might be correctly received. Nevertheless, the messages would concurrently use the same slots and this should be considered in the computation of $N_{b}$. This leads to the following relationship:

$P_{c o l} E\left[N_{c o l}\right]=P_{c s} N_{s}+P_{c h} \frac{N_{s}}{2}$.

Replacing the terms in Eq. (3), we obtain (remember that we consider $n_{i} \approx 2$ ):

$E\left[N_{b}\right] \approx E\left[n_{c}\right] N_{s}\left(1-P_{\text {exp }}-\frac{P_{c s}}{2}-\frac{P_{c h}}{4}\right)$.

In order to start transmitting its CAM in a slot $k$ of the beaconing period, node $V$ must not experience an expired beacon phenomenon. If this prerequisite is fulfilled, the first slot is uniformly chosen among the $N_{T}$ slots of the beaconing period, and therefore the probability of node $V$ beginning its transmission in slot $k$ is:

$P_{k}=\frac{1-P_{\text {exp }}}{N_{T}}=P_{\text {start }}$.

In the following, we will provide formulas for the two collision probabilities (with sensed and hidden terminals respectively) and the beacon expiration probability.

\subsection{Synchronised transmissions}

To help understand the impact of synchronised transmissions and calculate the probability of such an event, the representation shown in Fig. 2 is used. $C_{V}$ denotes the set of nodes that can be sensed by $V$. A formal definition in this case is:

$C_{V}=\left\{v_{i} \mid d\left(v_{i}, V\right) \leqslant S_{r}\right\}$

where $d\left(v_{i}, V\right)$ is the distance between nodes $V$ and $v_{i}$. Using the same notation as in the previous section, $\left|C_{V}\right|=n_{c}$.

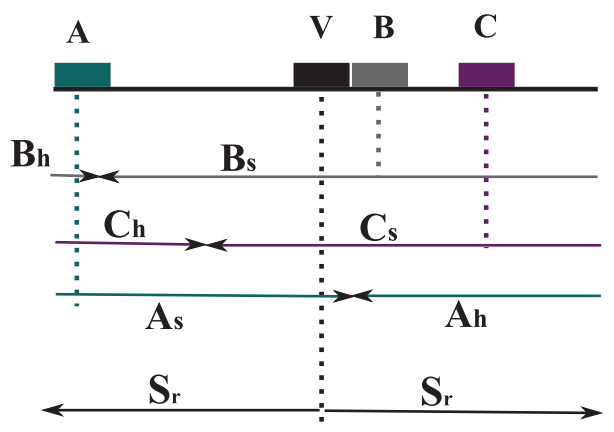

Fig. 2. Size variation of the zones occupied by $W_{s}$ and $W_{h}$ around vehicle $V$ as a function of its neighbour position. 
Choosing a vehicle $W \in C_{V}, W_{s}$ is defined as the set of nodes that can be sensed by both $V$ and $W$ $\left(W_{s}=C_{V} \cap C_{W}\right)$. From Fig. 2, we can notice that the size of the area occupied by the nodes in this set on the road map can vary between $S_{r}$ when the considered node is situated at the border of $V$ 's carrier sense range (e.g. node $A$ ), to $2 S_{r}$ when the neighbour is geographically close to node $V$ (e.g. node $B$ ).

We make the assumption of a unique carrier sense threshold, and we use the notation $n_{c s}=\left|W_{s}\right|$. In this case, knowing that $W$ and $V$ have $j$ common neighbours, the probability that node $W$ transmits a beacon without interfering with one of these neighbours at node $V$ is:

$P_{n o S j}=\left(P_{n o S} \mid n_{c S}=j\right)=\sum_{k=0}^{N_{T}-1} P_{\text {start }}\left(1-P_{\text {start }}\right)^{j}$

$P_{\text {noS } j}=N_{T} P_{\text {start }}\left(1-P_{\text {start }}\right)^{j}$

Assuming that $W$ is situated at distance $r$ from node $V$, with $-S_{r}<r<S_{r}$, the probability that a neighbour of $V$ belongs to $W_{s}$ is:

$\tau_{r}=1-\frac{|r|}{2 S_{r}}$

and the probability of having $n_{c s}=j$ when $r$ is known becomes:

$P_{j \mid r}=P\left(n_{c s}=j \mid r\right)=\left(\begin{array}{c}n_{c}-1 \\ i\end{array}\right) \tau_{r}^{j}\left(1-\tau_{r}\right)^{n_{c}-j-1}$.

Considering the symmetry of vehicle V's right and left side, the probability that $V$ does not experience synchronised transmissions from neighbours that can also sense each other can be written as:

$P_{n o S}=\int_{r=0}^{S_{r}} \frac{1}{S_{r}} \sum_{j=0}^{n_{c}-1} P_{n o S \mid j} P_{j \mid r} \mathrm{~d} r$.

After replacing the terms from Eqs. (5)-(7), this becomes:

$P_{\text {nos }}=\frac{N_{T} P_{\text {start }}}{S_{r}} \int_{0}^{S_{r}}\left(1-P_{\text {start }}+\frac{r P_{\text {start }}}{2 S_{r}}\right)^{n_{c}-1} \mathrm{~d} r$.

Finally, after solving and simplifying, we obtain:

$P_{\text {nos }}=\frac{2 N_{T}}{n_{c}}\left[\left(1-\frac{P_{\text {start }}}{2}\right)^{n_{c}}-\left(1-P_{\text {start }}\right)^{n_{c}}\right]$.

The probability of a synchronised transmission can now be calculated simply as

$P_{c s}=1-P_{\text {nos }}$

\subsection{Collisions with hidden nodes}

Using similar notations as in the case of synchronised transmissions, we define $W_{h}$ as the set of vehicles that can be sensed by $V$, but not by $W\left(W_{h}=C_{V} \backslash C_{W}\right)$. From Fig. 2, we can observe that the projection of this set on the road map covers an area varying from practically 0 for vehicles close to the tagged node (e.g. node $B$ ) to $S_{r}$ when a vehicle situated at the border of the carrier sense range is considered (e.g. node $A$ ).

While a synchronised transmission can only occur if two nodes start transmitting in the same slot, a collision with a hidden terminal at node $V$ takes place if any of the $i$ nodes belonging to $W_{h}$ begins a transmission during one of the $N_{s}$ slots occupied by $W$, or even in one of the $N_{s}-1$ preceding slots. Therefore, using the notation $n_{c h}=W_{h}$, the probability of avoiding such an event is:

$P_{\text {noH } \mid i}=\left(P_{\text {noH }} \mid n_{c h}=i\right)=\sum_{k=0}^{N_{T}-1} P_{\text {start }}\left(1-P_{\text {start }}\right)^{i\left(2 N_{s}-1\right)}$

$P_{n o H \mid i}=N_{T} P_{\text {start }}\left(1-P_{\text {start }}\right)^{i\left(2 N_{s}-1\right)}$.

Using a similar line of reasoning as for synchronised transmissions, the probability that a neighbour of node $V$ belongs to $W_{h}$ is:

$\phi_{r}=\frac{|r|}{2 S_{r}}$

and the probability of having $n_{c h}=i$ when $r$ is known can be written as:

$P_{i \mid r}=P\left(n_{c h}=i \mid r\right)=\left(\begin{array}{c}n_{c}-1 \\ i\end{array}\right) \phi_{r}^{i}\left(1-\phi_{r}\right)^{n_{c}-i-1}$.

The probability that a collision with a hidden node does not appear can be written as:

$P_{\text {noH }}=\int_{r=0}^{S_{r}} \sum_{i=0}^{n_{c}-1} \frac{1}{S_{r}} P_{n o H \mid i} P_{i \mid r} \mathrm{~d} r$

which, after substituting Eqs. (10)-(12), becomes:

$$
\begin{aligned}
P_{\text {noH }}= & \frac{N_{T} P_{\text {start }}}{S_{r}} \\
& \left.\times \int_{0}^{S_{r}}\left[1-r \frac{1-\left(1-P_{\text {start }}\right)^{\left(2 N_{s}-1\right)}}{2 S_{r}}\right)\right]^{n_{c}-1} \mathrm{~d} r .
\end{aligned}
$$

Finally,

the result after solving the integralis:

$\left.P_{\text {noH }}=\frac{2 N_{T} P_{\text {start }}}{n_{c}\left[1-\left(1-P_{\text {start }}\right)^{2 N_{s}-1}\right]}\left[1-\frac{1+\left(1-P_{\text {start }}\right)^{2 N_{s}-1}}{2}\right)^{n_{c}}\right]$

and the probability of a collision with a hidden nodes is simply:

$P_{c h}=1-P_{n o H}$.

\subsection{Beacon expiration probability}

The beaconing expiration probability $P_{\exp }$ is the last missing term in Eq. (3). In order to experience an expired message, a station first needs to find the channel busy when the beacon is passed from the network layer. This triggers a back-off of $b$, and the condition for the CAM to expire is that the node senses less than $b$ idle slots in the next beaconing period. The probability of this last event can be expressed as: 
$P_{\text {idle }}(b)=\sum_{j=0}^{b-1}\left(\begin{array}{c}N_{T} \\ j\end{array}\right)\left(1-P_{b}\right)^{j} P_{b}^{N_{T}-j}$.

Finally, assuming the backoff is uniformly chosen between 0 and $C W-1$, the expiration probability is:

$P_{\text {exp }}=P_{b} \sum_{b=1}^{C W} \frac{1}{C W} P_{\text {idle }}(b)$.

At this point, the system formed by Eqs. (2), (4), (8), (15) and (16) can be solved, and other results can be calculated afterwards.

\subsection{Numerical example}

In a network where all the nodes use a data rate of $6 \mathrm{Mb} / \mathrm{s}$ and a beaconing period of $100 \mathrm{~ms}$, a maximum of 150 messages with a size of 500 bytes each can be received by vehicle $V$. This scenario assumes perfect transmission synchronisation, and it is not possible for example in an IEEE 802.11 network, where an inter-frame space needs to be inserted after every message and the use of a random back-off leads to a non-zero collision probability. However, this perfect sequence of events is used in this section as a reference, helping us construct a similar example that can be studied using the framework detailed in the previous sections.

Following this line of reasoning, the VANET analysed below uses a beaconing period of 1500 slots, with a single transmission taking 10 slots, leading to the same network capacity of 150 messages during one period. It should be noticed that the size and the meaning of a slot are not the same as in the IEEE 802.11 case, where the time slot is calculated as the duration necessary for symbol transmission and signal propagation in the desired coverage area. In our model, a slot is simply the smallest time unit considered, which, under the assumption of a $10 \mathrm{~Hz}$ beaconing frequency, has a duration of $66.7 \mu \mathrm{s}$, much larger than the $13 \mu$ s slot time in IEEE 802.11. The relationship between the two slot values is difficult to determine, as every time the IEEE 802.11 timer is frozen, a DIFS period is added to the waiting time. It should be therefore noted that the actual values of $C W$ obtained using the analytical model should not be directly compared with the simulation results, as their significance is different.

Because our goal is to test the performance of the MAC protocol in medium to high density scenarios, the number of nodes sensed by vehicle $V, n_{c}$, is varied between 100 and 250 . While this final value might seem exaggerated, in a classical two-way highway with three lanes for each direction and a carrier sense range of $1 \mathrm{~km}$, as predicted for IEEE $802.11 \mathrm{p}$, this produces a density of $42 \mathrm{veh} / \mathrm{lane} / \mathrm{km}$, or an inter-vehicular distance of $24 \mathrm{~m}$, not uncommon in most urban areas for rush hour traffic. It is also important to notice that this scenario leads to a network load $\rho$ higher than the $\mathrm{CCH}$ capacity. This means that, necessarily, some of the messages either collide or expire.

The system of equations discussed in the previous sections is solved using an iterative method where $P_{b}$ is initially set to $P_{b o}=\min (1 ; \rho)$. The expiration and collision probabilities are computed using $P_{b o}$, and a new value for the busy slot probability $P_{b n}$ is also calculated. In the following iteration $P_{b o}$ takes the value of $P_{b n}$ and the process is repeated until $\left|P_{b n}-P_{b o}\right|<10^{-4}$.

The impact the contention window has on the collision probability is first discussed. Fig. 3 presents the probability of a synchronised transmission $\left(P_{c s}\right)$ at node $V$ for three different numbers of one-hop neighbours. This implies that two of $V$ 's neighbours, also situated inside each other's carrier sense range, begin transmitting at the same time, following the expiration of the back-off timer, or because the medium is idle when a safety message arrives from the network layer. From the figure, the behaviour of $P_{c s}$ can be divided in three phases. In the first phase, increasing the contention window has no impact whatsoever on the collision probability. However, the interval of the contention window over which this uniform behaviour can be observed decreases when the vehicular density increases, and it becomes negligible for $n_{c}=200$. In the second stage, the number of collisions decreases steadily, until reaching a minimum where the third phase, a slower increase, begins. It is noteworthy that, because we only show results for a contention window up to 450 , the third phase for $n_{c}=125$ is not visible in the figure, as it starts at a higher value of the contention window.

The same observations can be made in the case of collisions between hidden nodes, presented in Fig. 4. In this case, a collision is the result of concurrent transmissions by two of node $V$ 's neighbours that are hidden from one another. We can notice that the probability of a collision with a hidden terminal is much higher than the one of a synchronised transmission. However, we must point out that our model does not take into account the capture effect. In a real scenario, an important number of these messages would be received by node $V$, especially in the case of collisions between hidden nodes, where the difference between the power level of the colliding messages is very often large enough to allow the reception of one of them. For example, experiments on the WINLAB ORBIT test-bed have shown that, in a high density scenario, the physical layer capture property of IEEE 802.11 devices recovers a frame in $50 \%$ of the encountered collisions [32].

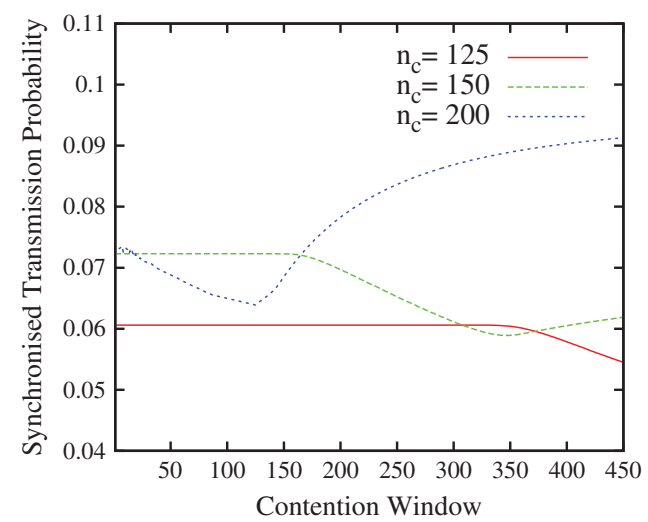

Fig. 3. Probability that node $V$ experiences a collision between two nodes situated inside each other's carrier sense range (synchronised transmissions) as a function of the contention window. 


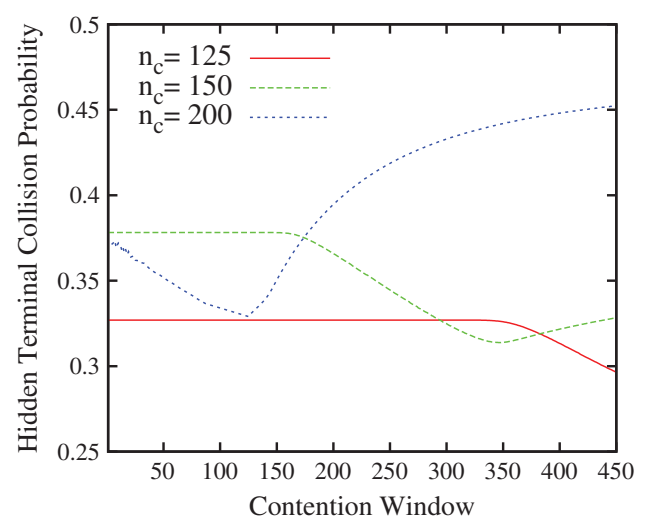

Fig. 4. Probability that node $V$ experiences a collision between two nodes situated outside each other's carrier sense range (hidden nodes) as a function of the contention window.

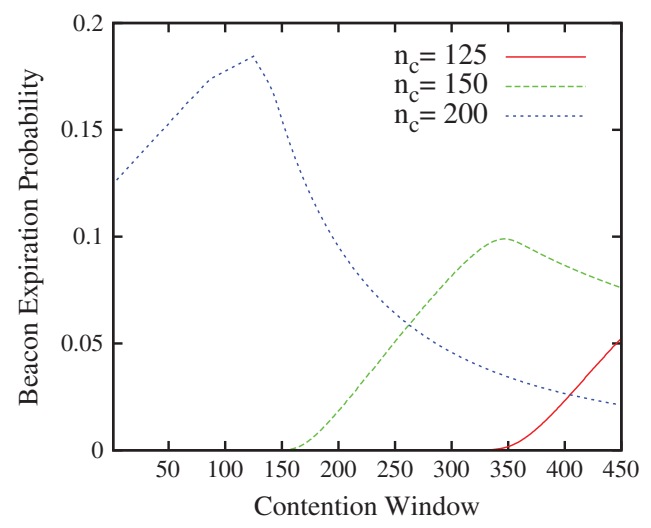

Fig. 5. Beaconing expiration probability as a function of the contention window for different vehicular densities.

These results can be better understood by observing Fig. 5, where the beaconing expiration probability under the same scenario is depicted. Initially, the contention window is too small to produce any expiration. This translates in the phase with the highest collision probability in Figs. 3 and 4 . When $C W_{\min }$ becomes large enough, more and more messages begin expiring, which reduces the network load and, with it, the collision probability. However, these modifications also have an impact on the probability of sensing a busy slot. Under the influence of an increased number of expired messages, $P_{b}$ slowly decreases, the reduced number of collisions not being able to fill up all the extra-bandwidth obtained from reducing the channel load. However, from Eq. (16), it can be noticed that the expiration probability $\left(P_{\text {exp }}\right)$ also depends on $P_{b}$. Reducing $P_{b}$ under a certain threshold leads to a reduction in $P_{\exp }$ and therefore to the third phase appearing on all these figures.

Because the beaconing reception probability takes into account both expired and collided messages, a balance needs to be found between the two quantities. Achieving this trade-off is not a simple task considering the fact that a collision is a local event, that only occurs at some of the neighbours, whereas an expired beacon is lost for all the possible receivers. Fig. 6 tries to portray the role played by the contention window on the number of received beacons, showing the reception probability (in fact the probability that a beacon does not expire and does not experience any kind of collision) for different network loads.

These results show that initially the gain from the avoided collisions compensates the loss produced by the expired messages, resulting in a sharp increase of the reception probability. After a certain point, the number of expired beacons begins to prevail and the reception probability starts a slower, but steady, decrease. When $P_{\exp }$ goes beyond its peak (and the number of collisions starts increasing again), the reception probability goes through another phase, where its value remains almost constant. Finally, when the expiration probability becomes low enough, the effect of the collisions is again predominant and the number of total receptions starts decreasing once more.

Another interesting result that can be observed from Fig. 6 is that the optimal value of the contention window decreases when the network load becomes higher. This behaviour is the opposite of the one described by the Bianchi relationship [6], where the optimal $C W_{\min }$ is in direct proportionality with the number of contending nodes.

\section{Reverse back-off mechanism}

The results presented in the previous section point out that using an improper value for the contention window can result in a significant drop in MAC layer performance. Despite this, both WAVE and ETSI ITS architectures are based on the IEEE 802.11e access method and use relatively low values for $C W_{\min }$ (between 3 and 15, depending on the access class). However, these values belong to the interval resulting in the worst beaconing reception probability, and a different approach is clearly needed in order to improve the MAC layer performance on the VANET CCH.

The new back-off mechanism we propose does not try to achieve optimality through an estimation of the number of neighbours, although this would be perhaps possible using the insight gained from the analytical study

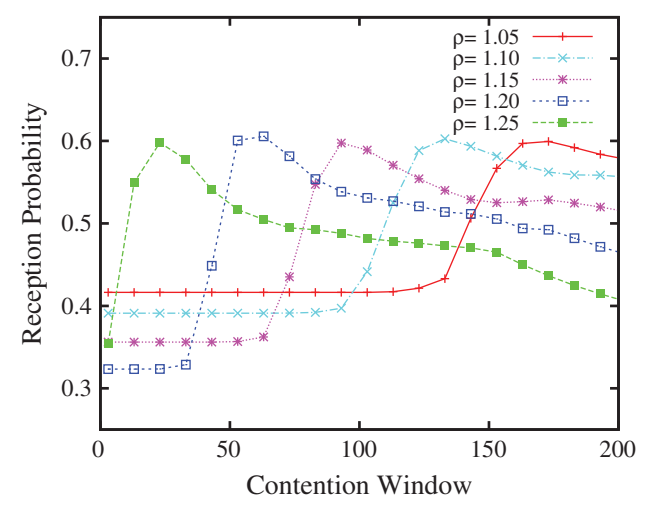

Fig. 6. Reception probability for safety beaconing as a function of the contention window for different values of the network load. 
discussed above. Instead, it is based on the observation that the most successful proposals in this field (e.g. IEEE 802.11 binary exponential back-off, optimal-CSMA contention aggressiveness) rely on internal information, accurate and easy to interpret. However, as already discussed, the state of the queues is not relevant for vehicular safety communications, and collisions remain undetectable in this broadcast environment. Another parameter is therefore needed in the computation of the contention window. Following the analytical results presented in the previous section, the optimal $C W$ in a VANET must find the correct balance between collided and expired messages. While the former cannot be used, the latter can be detected with no effort and they can form the basis of a back-off mechanism.

There are two ideas behind the proposed mechanism:

- A larger contention window than the one proposed in IEEE $802.11 \mathrm{p}$ is needed in order to cope with medium and high vehicular densities.

- An expired beacon produces important consequences, because the information it carries will not be received by any neighbour, and therefore needs to be counterbalanced by an increased priority of the next beacons produced by that vehicle.

Therefore, instead of beginning with a small $C W_{\min }$ that would be increased after every failed transmission, as in IEEE 802.11 BEB, the reverse back-off mechanism we designed starts with a fairly large initial contention window $\left(C W_{i}\right)$ and decreases it for every expired beacon. While different approaches could be taken when $C W$ is increased or decreased, the results shown in this paper consider $C W$ is halved after every expired CAM and it returns to the original value $C W_{i}$ after $n_{t}=1$ transmitted beacons.

While the properties of the reverse back-off mechanism (RB) will be studied in more detail in the next section, some observations can already be made:

- Similarly to oCSMA [20], the reverse back-off mechanism reduces the aggressiveness of the nodes under normal functioning conditions. By using a larger interval for the back-off time, RB is able to reduce the number of collisions.

- In a high density vehicular network, the control channel cannot accommodate the entire network load. It is obvious that a part of the messages will be lost in such a scenario, either through a collision or following an expiration. In this case, a MAC protocol should not only focus on reducing the losses, but should also consider the distribution of these losses, as a vehicle losing several beacons in a row represents a higher danger than several vehicles losing each one beacon. By using a low value for the contention window, as currently proposed in IEEE $802.11 p$, most of the losses will be the result of a collision. However, collisions are unpredictable events, and the MAC protocol cannot control the distribution of the losses in this case. The RB mechanism, on the other hand, increases the proportion of expired messages whose distribution is much easier to control in a vehicular context.
- It might be assumed that, in a sparse network, the reverse back-off mechanism would unnecessarily increase the delay for safety messages. However, it must be pointed out that, under low vehicular traffic, a node has a high probability to detect the $\mathrm{CCH}$ idle and transmit immediately, without going through a back-off stage.

\section{Mechanism evaluation}

As the reverse back-off mechanism is designed for highly congested vehicular networks, an experimental evaluation is simply prohibitive. In these conditions, we decided to conduct an in depth simulation study, using models that match reality as close as possible. This section begins with a description of the simulation framework used in this study. The simulation scenario and different parameters are then presented, before a detailed discussion regarding the obtained results.

\subsection{Simulation framework}

The simulation of vehicular networks presents significant challenges when compared with classical MANETs. The large scale of VANETs or the need for new mobility models and radio propagation models are just a few of the problems needing to be solved.

All the simulations discussed in this paper were conducted using the Java in Simulation Time (JiST) environment [33]. JiST is a general purpose discrete-event simulator built in Java. A framework dedicated to wireless network simulation, the Scalable Wireless Ad hoc Network Simulator (SWANS), is also available on top of JiST. The JiST/SWANS platform and models (including the IEEE 802.11 model) have been extensively tested by Weingartner et al. [34] and the framework is widely used in the study of vehicular networks.

Realistic vehicular mobility is provided by the Street Random Waypoint (STRAW) package, developed at the University of Ohio [35] and integrated in JiST/SWANS. The software creates real topologies from US Census Bureau's TIGER data files and it accurately describes stop signs and traffic lights. STRAW contains three different modules. One of them is dedicated to intra-segment mobility, the second one to inter-segment mobility and the last one to route planning. The vehicular movement is described using a car-following model, which is designed using a leader-follower logic. If the distance between a car and the next vehicle on the lane is larger than a certain threshold, the car movement is described only by its maximum speed and acceleration. When the distance to the vehicle in front decreases under the threshold, the follower starts decelerating until it matches the speed of the next car. When a car accelerates, its follower will do the same thing until it reaches its own maximum speed.

\subsection{Simulation scenario}

The IEEE WAVE architecture includes a switching mechanism between the $\mathrm{CCH}$ and $\mathrm{SCH}$, in order to allow singleradio devices to benefit from both safety and non-safety 
applications. On the other hand, the ETSI architecture assumes the existence of multiple radios, one of which would always be listening or transmitting on the control channel. In our study, we consider a dedicated radio for the $\mathrm{CCH}$ is used. All the MAC and PHY layer parameters are set according to the IEEE $802.11 \mathrm{p}$ standard [2], and the simulator specific parameters are summarised in Table 1.

We use a fixed beaconing frequency of $10 \mathrm{~Hz}$ and a beacon size of 500 bytes that includes the payload, the different headers and the security overhead. These values are in line with those predicted by the European research projects in this field [7].

To remove any bias introduced by a particular topology, all the simulations we discuss were repeated using three different maps with a size of $5 \mathrm{~km} \times 5 \mathrm{~km}$. The total road length in these three cases varied from $11.3 \mathrm{~km}$ to $18.9 \mathrm{~km}$. A number of 30 runs, using different random seeds, were conducted for each road topology and for each parameter setting. The initial positioning of the vehicles on the map was random, but a warming period of 10 min was used at the beginning of every simulation. During this time, the communication module was turned off and only the vehicular mobility was simulated, in order to eliminate any transient phase introduced by the mobility. After these $10 \mathrm{~min}$, the safety application was launched on every node and the communications began for another $10 \mathrm{~min}$. However, only the messages transmitted in the final 5 min, when the system already reached a steady-state, were used to compute the statistics. In order to avoid boundary effects, the results were always gathered only from a central region of the map. The vehicles outside this area of interest participated in the communication, but their receptions were not taken into account in the final results. Therefore, statistics coming from vehicles situated at less than $1 \mathrm{~km}$ from the border were not used when computing the results.

As already mentioned, the radio propagation model is a major challenge in VANET simulation. The model used in this study is based on the modified shadowing model for vehicular environments proposed by Dhoutaut et al. [36], where the fast fading factor is not completely random, but depends on the vehicular density. This model is based on the idea that more vehicles bring more reflections for the signal and, hence, a higher level of interference. The idea is backed-up by experimental results detailed in [36]. Fig. 7 shows the beaconing reception probability (without any interference from other nodes) with increasing distance from the transmitter under sparse and dense traffic when this propagation model is used in our simulator. As it can be noticed, the reception probability decreases at higher distances when the vehicular density increases, modelling the increased number of reflections and multi-paths.

Table 1

Simulation parameters.

\begin{tabular}{ll}
\hline Parameter & Value \\
\hline Antenna gain & $0 \mathrm{dBm}$ \\
Receiver sensitivity & $-91 \mathrm{dBm}$ \\
SINR required to decode a message & $10 \mathrm{~dB}$ \\
Ambient noise level & $-95 \mathrm{dBm}$ \\
Data rate & $6 \mathrm{Mb} / \mathrm{s}$ \\
\hline
\end{tabular}

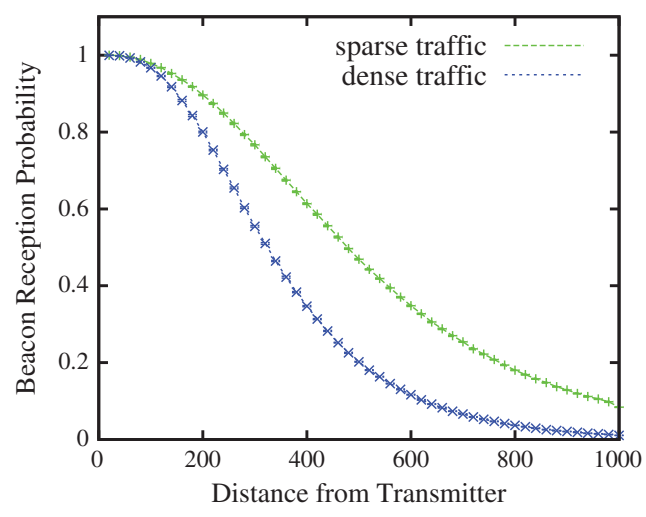

Fig. 7. Reception probability for safety beaconing with increasing distance from the transmitter using the radio propagation model from [36] under different vehicular densities.

Several important observations need to be made at this point. First of all, the profile of the beaconing reception probability in Fig. 7 corresponds very well to experimental studies like the one in [14], where the large area occupied by grey zones (zones with a reception probability between $20 \%$ and $80 \%$ ) has been noticed. Second, although the range of IEEE $802.11 \mathrm{p}$ radios can be close to $1 \mathrm{~km}$ when the maximum transmission power is used, our scenario is far from this point. This is because our study is focused on scenarios when a power level close to the minimum is already used, meaning that transmission power control mechanisms have already reached their limit and a complementary mechanism is needed. Finally, while the accuracy of the radio propagation model we used was confirmed in the case of highway scenarios, simulating realistic urban scenarios still remains a difficult task, as it would require an accurate knowledge of the topology and the alterations introduced by buildings. As a consequence, the results presented throughout this study are issued from simulations using maps of sub-urban areas that include multiple types of roads. Another important observation at this point is that the different roads appearing on the maps have different properties and can accommodate different traffic densities. In the remaining of this paper, we use the mean vehicular density over the entire map as a parameter, but it is noteworthy that this is not a uniform density and that in our simulations major road arteries generally present a higher density than secondary roads.

\subsection{Results}

The analytical model described in the previous section predicts fundamental differences in the way the contention window impacts the reception of beacons when compared with regular broadcast traffic, where the messages cannot expire. Simulation results presented in Fig. 8 show the optimal value of the contention window for regular broadcast (no expiration) and beaconing (with expiration) scenarios. In both cases, we denote as optimal CW the contention window that results in the highest packet reception ratio. The results confirm the property uncovered analytically in Section 3, showing that the value of the 


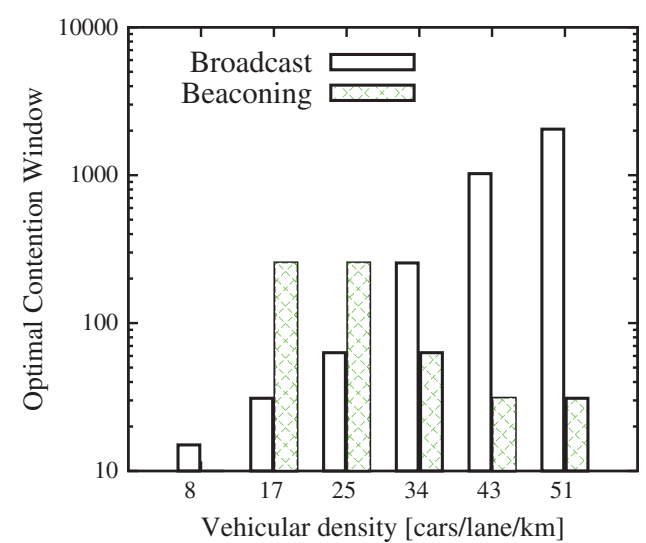

Fig. 8. Optimal contention widow for pure saturated broadcast and beacon messages under different vehicular densities (please notice the log-scale of the $y$-axis). The optimal value for the beaconing scenario with a density of $8 \mathrm{cars} / \mathrm{lane} / \mathrm{km}$ could not be determined because most $\mathrm{CW}$ values produces similar results for the reception ratio.

optimal CW behaves totally different when a threshold is set on the lifetime of the messages. We can notice that the results for the broadcast scenario follow the predictions of the Bianchi model [6], and the optimal CW increases with the number of nodes. On the other hand, when beacons are considered, the best contention window takes a smaller value when the vehicular density increases, confirming our analytical results.

This totally opposite behaviour can be easily explained. In the case of saturated broadcast, the reason behind a lost message is either a collision or a radio propagation problem. When the number of vehicles increases, the contention window must follow the trend in order to reduce the collision probability. The fact that beacons can expire adds another possible reason for a lost message, and we should not forget that an expired CAM is lost for all the neighbours, unlike in the case of a collision. Therefore, in this second case, when the number of nodes in the network increases, the contention window must be decreased in order to reduce the expiration probability to an acceptable value.

The beaconing reception probability for different values of the contention window can be better analysed in Fig. 9, where three different vehicular densities are considered. As the reception probability depends on the distance to the transmitter, we decided to focus on receivers situated in a $100 \mathrm{~m}$ range, a region where safety messages can be critical. It can be noticed that, in all the cases, the evolution of the reception probability is similar: a relatively sharp increase until an optimal value is reached, a zone where the reception probability remains constant, followed by a slower, but steady decrease. As in the previous results, we can see that the optimal value of $C W$ is smaller when the node density increases. An important observation in this figure is that the value $C W=7$ currently proposed in the standard for CAM messages is far from optimal, and a $10 \%$ improvement can be obtained by a correctly parametrised contention window.

Just like in the case of the BEB mechanism, the performance of the reverse back-off mechanism depends on the

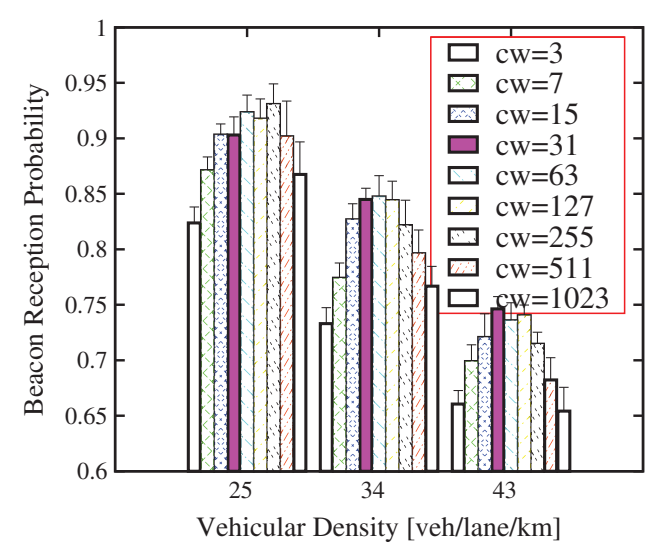

Fig. 9. Beaconing reception probability at $100 \mathrm{~m}$ from the transmitter for different values of the contention window under three different vehicular densities.

initial value of the contention window $\left(C W_{i}\right)$. After studying the impact of different $C W_{i}$ values on the RB mechanism, we decided to set this parameter to 127 (the $C W$ values are usually calculated as $2^{m}-1$, so in our case $m=7$ ), although all the values between 60 and 200 lead to similar results. Fig. 10 shows the reception probability of the RB mechanism with $C W_{i}=127$ under different vehicular densities. These results are compared with those obtained by using the current IEEE $802.11 \mathrm{p}$ standard (a fixed $C W=7$ ) and by the best fixed value of the contention window for every scenario. We can notice that the RB mechanism is close to the optimal $C W$ value, bringing an improvement of about $10 \%$ over the current standard when the vehicular density is high. However, it must be pointed out that the optimal value of the contention window depends on the vehicular density, as discussed above. On the other hand, the reverse back-off mechanism uses the same set of parameters regardless the number of nodes in the network.

Moreover, an even more significant difference can be noticed in Fig. 11, depicting the number of consecutive beacons lost between pairs of vehicles situated at less than

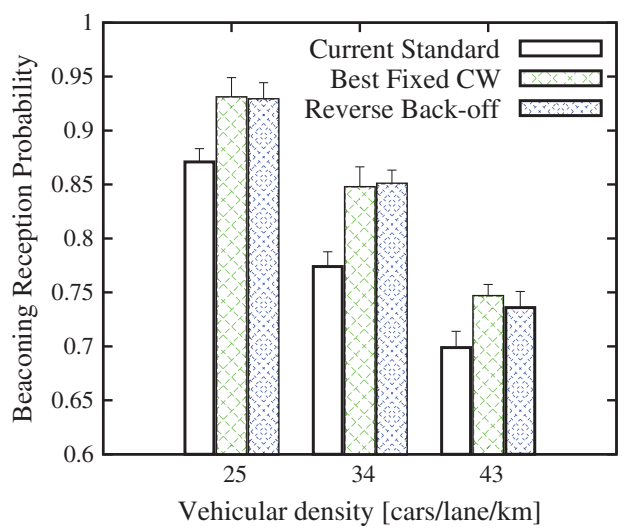

Fig. 10. Beaconing reception probability at $100 \mathrm{~m}$ from the transmitter using the CW currently proposed in the IEEE $802.11 \mathrm{p}$ standard, the best fixed contention window and the reverse back-off mechanism under different vehicular densities. 
$100 \mathrm{~m}$ from one another. This can be seen as a measure of the awareness a vehicle has on the state of the safety critical area around it, as more messages lost in a row reduce the quality of the estimation a node has on the location of the surrounding vehicles. As we use a fixed beaconing frequency, this metric can also be interpreted in terms of the time duration between the reception of two beacons from a given neighbour. For this, it is sufficient to multiply the number of consecutive lost beacons with the beaconing period (100 ms in our case). While, as seen above, the number of losses is similar for the optimal fixed $C W$ and the reverse back-off mechanism, the distribution of these losses is highly different. Using the reverse back-off, there are $18 \%$ more cases when a node misses between 1 and 9 consecutive beacons from one of its neighbours than when using a fixed contention window. The probability of losing between 10 and 20 consecutive beacons is similar in both cases ( $2 \%$ less cases for the reverse back-off). Finally, an event where more than 20 consecutive beacons are lost appears $40 \%$ less often when using the new back-off mechanism. This metric is essential for safety applications, where invisible neighbours represent a danger and their existence must be minimised. Increasing the priority of a node following an expired beacon, as proposed in the reverse back-off mechanism, manages to reduce the probability of such ghost nodes, a very important property in a vehicular environment.

To better understand the behaviour of the RB mechanism, we also show the distribution of the reasons that can lead to a lost safety message at different distances from the sender when using the current version of the standard (Fig. 12) and the reverse back-off mechanism (Fig. 13). For example, at $40 \mathrm{~m}$ from the sender, more than $90 \%$ of the messages lost using standard IEEE 802.11p are the consequence of simultaneous transmissions with nodes located inside the carrier sense range, and less than $10 \%$ are due to collisions with hidden nodes. On the other hand, when the proposed mechanism is used, the loss distribution changes, and the impact of synchronised transmissions becomes less significant. This is due to the higher value of the contention window, which reduces the number of

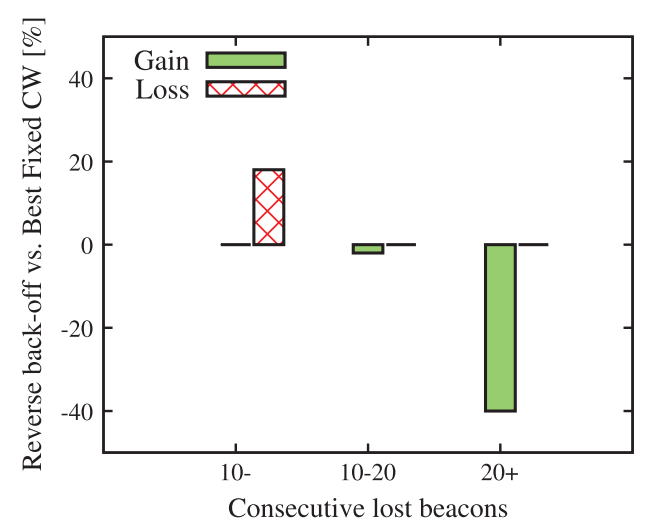

Fig. 11. Comparative representation of the number of consecutive beacons lost between pairs of nodes situated at less than $100 \mathrm{~m}$ from each other for a density of $43 \mathrm{veh} / \mathrm{lane} / \mathrm{km}$.

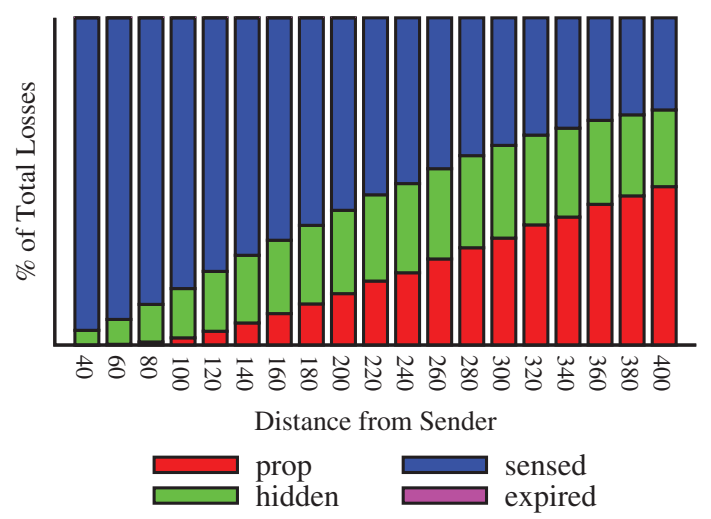

Fig. 12. Distribution of the reasons for a lost message at different distances from the sender without using the reverse back-off mechanism.

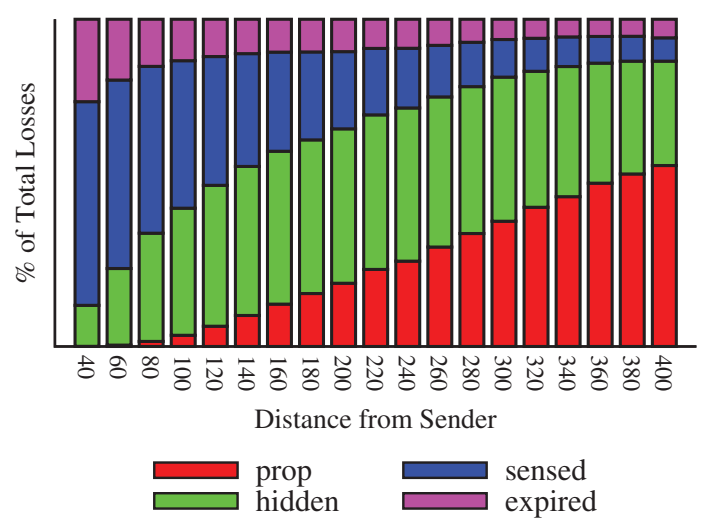

Fig. 13. Distribution of the reasons for a lost message at different distances from the sender using the reverse back-off mechanism.

collisions, but introduces more expired messages (not a significant loss reason in Fig. 12).

From the results presented in this section, we can conclude that the RB mechanism increases the performance of IEEE $802.11 \mathrm{p}$ in dense vehicular networks, especially in the immediate neighbourhood of the vehicles, where safety information has the highest relevance. Moreover, the reverse back-off does not only increase the beaconing reception probability, but it also modifies the distribution of the lost messages and their reasons.

\section{Conclusion}

The design of an efficient and scalable MAC layer is an essential step towards the creation of a safety vehicular network on our roads. This paper is focused on IEEE $802.11 \mathrm{p}$, the technology widely considered as the best choice in this context, and more precisely on the back-off mechanism proposed in the standard. We show that the value currently assigned to the contention window is far from being optimal, especially when the vehicular density increases. Using an analytical framework and an extensive simulation study, we argue that expired messages should not be feared, as, in a reduced number, they can actually 
improve the performance of safety applications. The reverse back-off mechanism uses these easily detectable expired messages to adjust the contention window and keeps their distribution under control. Designed particularly for the VANET control channel, this mechanism alleviates the scalability problem of the current standard and is totally compatible with other congestion control mechanisms currently under standardisation.

\section{References}

[1] A. Minino, J. Xu, K. Kochanek, Deaths: preliminary data for 2008, U.S Natl. Vital Stat Rep 59 (2) (2010)

[2] The Institute of Electrical and Electronics Engineers, IEEE Standard for Information Technology - Telecommunications and Information Exchange Between Systems - Local and Metropolitan Area Networks - Specific Requirements. Part 11: Wireless LAN Medium Access Control (MAC) and Physical Layer (PHY) Specifications - Amendment 6: Wireless Access in Vehicular Environments, July 2010

[3] S. Xu, T. Saadawi, Does the IEEE 802.11 MAC protocol work well in multihop wireless ad hoc networks?, IEEE Commun Mag. 39 (6) (2002) 130-137.

[4] T. Kosch, I. Kulp, M. Bechler, M. Strassberger, B. Weyl, R. Lasowski, Communication architecture for cooperative systems in Europe, IEEE Commun. Mag. 47 (5) (2009) 116-125.

[5] The European Telecommunications Standards Institute, ETSI TS 102 687 v1.1.1 - Intelligent Transport Systems (ITS) - Decentralized Congestion Control Mechanisms for Intelligent Transport Systems Operating in the $5 \mathrm{GHz}$ Range - Access Layer Part, July 2011.

[6] G. Bianchi, Performance analysis of the IEEE 802.11 distributed coordination function, IEEE J. Sel. Areas Commun. 18 (3) (2000) 535 547.

[7] P. Papadimitratos, A. De La Fortelle, K. Evenssen, R. Brignolo, S. Cosenza, Vehicular communication systems: enabling technologies applications, and future outlook on intelligent transportation, IEEE Commun. Mag. 47 (11) (2009) 84-95.

[8] The European Telecommunications Standards Institute, ETSI TS 102 637-1 v1.1.1 - Intelligent Transport Systems (ITS) - Vehicular Communications - Basic Set of Applications - Part 1: Functional Requirements, September 2010.

[9] R. Oliveira, L. Bernardo, P. Pinto, The influence of broadcast traffic on IEEE 802.11 DCF networks, Comput. Commun. 32 (2) (2009) 439 452.

[10] R. Schmidt, T. Leinmuller, E. Schoch, F. Kargl, G. Schafer, Exploration of adaptive beaconing for efficient intervehicle safety communication, IEEE Netw. 24 (1) (2010) 14-19.

[11] C. Sommer, O. Tonguz, F. Dressler, Traffic information systems: efficient message dissemination via adaptive beaconing, IEEE Commun. Mag. 49 (5) (2011) 173-179.

[12] T. Tielert, D. Jiang, H. Hartenstein, L. Delgrossi, Joint power-rate congestion control optimizing packet reception in vehicle safety communication, in: Proceedings of the 10th ACM International Workshop on Vehicular Ad Hoc Networks (VANET 2013), Taipei, June 2013, pp. 51-60.

[13] Y. Mertens, M. Wellens, P. Mahonen, Simulation-based performance evaluation of enhanced broadcast schemes for IEEE 802.11-based vehicular networks, in: Proceedings of the 67th IEEE Vehicular Technology Conference (VTC Spring 2008), Singapore, 2008, pp. 3042-3046.

[14] F. Bai, D. Stancil, H. Krishnan, Toward understanding characteristics of dedicated short range communications (DSRC) from a perspective of vehicular network engineers, in: Proceedings of the 16th ACM Annual International Conference on Mobile Computing and Networking (MOBICOM 2010), Chicago, 2010, pp. 329-340.

[15] M. Sepulcre, J. Mittag, P. Santi, H. Hartenstein, J. Gozalvez, Congestion and awareness control in cooperative vehicular systems, Proc. IEEE 99 (7) (2011) 1260-1279.

[16] R. Stanica, E. Chaput, A.-L. Beylot, Physical carrier sense in vehicular ad-hoc networks, in: Proceedings of the 8th IEEE International Conference on Mobile Ad-Hoc and Sensor Systems (MASS 2011) Valencia, 2011, pp. 1-10.
[17] F. Cali, M. Conti, E. Gregori, Dynamic tuning of the IEEE 802.11 protocol to achieve a theoretical throughput limit, IEEE/ACM Trans. Network. 8 (6) (2000) 785-799.

[18] M.-S. Kim, D.-H. Kwon, Y.-J. Suh, Adaptive window mechanism for the IEEE 802.11 MAC in wireless ad hoc networks, in: Proceedings of the 19th International Conference on Information Networking (ICOIN 2005), Jeju, 2005, pp. 31-40.

[19] M. Heusse, F. Rousseau, R. Guillier, A. Duda, Idle sense: an optimal access method for high throughput and fairness in rate diverse wireless LANs, in: Proceedings of the ACM SIGCOMM Conference on Data Communication (SIGCOMM 2005), Philadelphia, 2005, pp. 121-132.

20] L. Jiang, J. Walrand, A distributed CSMA algorithm for throughput and utility maximization in wireless networks, IEEE/ACM Trans. Network. 18 (3) (2010) 960-972.

[21] T. Kim, J. Ni, R. Srikant, N. Vaidya, On the achievable throughput of CSMA under imperfect carrier sensing, in: Proceedings of the 30th IEEE Annual International Conference on Computer Communications (INFOCOM 2011), Shanghai, 2011, pp. 1674-1682.

[22] D. Rawat, G. Yan, D. Popescu, M. Weigle, S. Olariu, Dynamic adaptation of joint transmission power and contention window in VANET, in: Proceedings of the IEEE 70th Vehicular Technology Conference (VTC Fall 2009), Anchorage, 2009, pp. 1-5.

[23] M. Di Felice, A. Ghandour, H. Artail, L. Bononi, On the impact of multi-channel technology on safety-message delivery in IEEE 802.11p-1609.4 vehicular networks, in: Proceedings of the IEEE 21st International Conference on Computer Communications and Networks (ICCCN 2012), Munich, 2012, pp. 1-8.

[24] M. Di Felice, L. Bedogni, L. Bononi, Group communication on highways: an evaluation study of geocast protocols and applications, Ad Hoc Netw. 11 (3) (2013) 818-832.

[25] A. Ghandour, M. Di Felice, H. Artail, L. Bononi, Dissemination of Safety Messages in IEEE 802.11p-WAVE Vehicular Network: Analytical Study and Protocol Enhancements, Pervasive and Mobile Computing, March 2013, http://dx.doi.org/10.1016 j.pmcj.2013.03.003.

[26] X. Ma, X Chen, H Refai, Unsaturated performance of IEEE 802.11 broadcast service in vehicle-to-vehicle networks, in: Proceedings of the 66th IEEE Vehicular Technology Conference (VTC Fall 2007), Baltimore, 2007, pp. 1957-1961.

27] A. Vinel, V. Vishnevsky, Y. Koucheryavy, A Simple analytical model for the periodic broadcasting in vehicular ad-hoc networks, in: Proceedings of the IEEE 4th Broadband Wireless Access Workshop (BWA 2008), New Orleans, 2008, pp. 1-5

[28] K.A. Hafeez, L. Zhao, Z. Liao, B.N. Ma, Performance analysis of broadcast messages in VANETs safety applications, in: Proceedings of the 53rd IEEE Global Telecommunications Conference (GlobeCom 2010), Miami, 2010, pp. 1-5

[29] C. Han, M. Dianati, R. Tafazolli, R. Kernchen, X. Shen, Analytical study of the IEEE 802.11p MAC sublayer in vehicular networks, IEEE Trans. Intell. Transport. Syst. 13 (2) (2012) 873-886.

[30] C. Campolo, A. Molinaro, A. Vinel, Y. Zhang, Modeling prioritized broadcasting in multichannel vehicular networks, IEEE Trans. Veh. Technol. 61 (2) (2012) 687-701.

[31] M. Di Felice, L. Bedogni, L. Bononi, DySCO: a dynamic spectrum and contention control framework for enhanced broadcast communication in vehicular networks, in: Proceedings of the 10th ACM International Symposium on Mobility Management and Wireless Access (MobiWac 2012), Paphos, 2012, pp. 97-106.

[32] K. Ramachandran, M. Gruteser, R. Onishi, T. Hikita, Experimental analysis of broadcast reliability in dense vehicular networks, IEEE Veh. Technol. Mag. 2 (4) (2007) 26-32.

[33] R. Barr, Z. Haas, R. van Renesse, JiST: an efficient approach to simulation using virtual machines, Softw. - Practice Exp. 35 (2005) 539-576.

[34] E. Weingartner, H. von Lehn, K. Wehrle, A performance comparison of recent network simulators, in: Proceedings of the 44th IEEE International Conference on Communications (ICC 2009), Dresden, May 2009, pp. 1-5.

[35] D. Choffnes, E. Bustamante, An integrated mobility and traffic model for vehicular wireless networks, in: Proceedings of the 2nd ACM International Workshop on Vehicular Ad Hoc Networks (VANET 2005), Cologne, September 2005, pp. 69-78.

[36] D. Dhoutaut, A. Regis, F. Spies, Impact of radio propagation models in vehicular ad hoc networks simulations, in: Proceedings of the 3 rd ACM International Workshop on Vehicular Ad Hoc Networks (VANET 2006), Los Angeles, September 2006, pp. 40-49. 


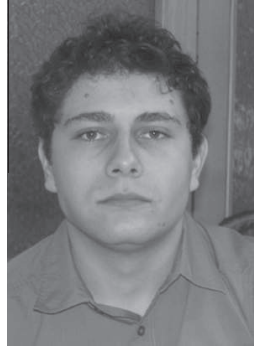

Razvan Stanica is an associate professor at INSA Lyon and a research scientist with the INRIA UrbaNet team of the Centre of Innovation in Telecommunications and Integration of Services (CITI). He obtained a Master in Engineering degree and a Ph.D. in computer science, both from the National Polytechnic Institute of Toulouse (INPT), France, in 2008 and 2011 respectively. He also obtained a Master in Engineering degree from the Polytechnic University of Bucharest, Romania, in 2008. His research interests include wireless mobile networks, with a special focus on vehicular communications and intelligent transportation systems.

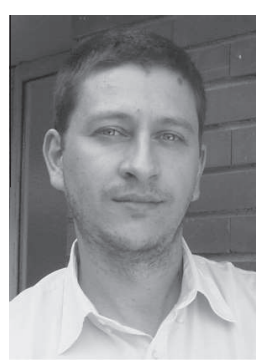

Emmanuel Chaput has received an engineering degree in computer science from ENSEEIHT, Toulouse (France) in 1992 and a Ph.D. degree in computer networks from the Université Paul Sabatier, Toulouse in 1998. He is now assistant professor (maître de conférences) at the Telecommunication and Network Department INPT/ENSEEIHT and member of the IRT team (Network and Telecommunication Engineering) CNRS/IRIT. His current research interests include IP over satellite networks architecture, but also vehicular communications. Within the scope of such application areas, he focuses on network architecture and resource allocation.

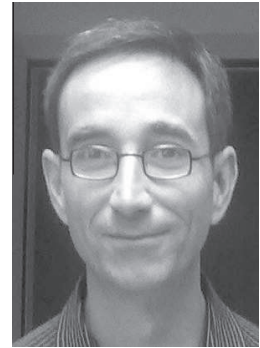

André-Luc Beylot received the Engineer degree from the Institut d'Informatique d'Entreprise, Evry, France, in 1989 and the Ph.D. degree in computer science from the University of Paris VI, Paris, France, in 1993. In January 2000, he received the Habilitation á Diriger des Recherches from the University of Versailles, France. From 1993 to 1995, he worked as a Research Engineer at the Institut National des Télécommunications, Evry, and from 1995 to 1996 at C.N.E.T. (France Telecom Research and Development), Rennes. From September 1996 to August 2000, he was an Assistant Professor at PriSM Laboratory, University of Versailles. Since September 2000, he has been a Professor at the Telecommunication and Network Department, National Polytechnics Institute of Toulouse (INPT/ENSEEIHT). His research interests are with the performance evaluation of communication networks, especially with regard to mobile, satellite, and vehicular networks. 